\section{Lifetime history of suicide attempts is associated with poorer social skills in patients with bipolar disorder type I}

\section{Tentativas de suicídio ao longo da vida associadas com pobre habilidade social em pacientes com transtorno bipolar tipo I}

Dear Editor,

Lifetime history of suicide attempts is frequently associated with bipolar disorder (BD) and can be associated with some risk factors, such as severity of depressive symptoms, impulsivity, hopelessness and family conflicts. ${ }^{1}$ Even during remission, patients with bipolar disorder can present social skill deficits. Rocca et al. showed that euthymic bipolar patients (BP) presented lower scores on items that assessed "conversation and social self-confidence" and "self-exposition to unknown people and new situations" on the Social Skills Inventory. ${ }^{2}$

However, there are no studies to evaluate social skills in bipolar patients with a past history of suicide attempts.

We studied a group of 28 euthymic outpatients with bipolar disorder type I: 12 with a history of suicide attempt, and 16 nonattempters. The groups of patients were compared with 31 healthy controls in a social skills measure. All participants were assessed using the Brazilian Social Skills Inventory, a self-report questionnaire. ${ }^{3}$

Patients with a history of suicide attempts presented lower IHS scores for the domains that assessed conversational skills and social self-confidence (domain 3: $\mathrm{p}<0.001$ ), social openness to new people and situations (domain $4: p=0.001$ ), self-control of aggressiveness and individual reactions to aversive stimuli that require the management of anger and aggressiveness (domain 5: $\mathrm{p}=0.041$ ) when compared to healthy controls and bipolar patients without previous suicide attempts.

Our results suggest that BPI outpatients with a history of suicide attempts present inhibited behaviour in relation to other people and their environment. They have difficulties to face situations that involve interacting with new people and they have to make efforts to control aggressiveness. Further studies may verify if these social skills deficits are associated with other risk factors that may lead to higher suicidality in bipolar patients.

This study was approved by the local ethics review committee and written informed consent was obtained from all participants (process $\left.n^{\circ} .0146 / 07\right)$.

Cristiana Castanho de Almeida Rocca, Luciana Gerchmann

Psychology and Neuropsychology Units, Institute of Psychiatry, Universidade de São Paulo (USP)

School of Medicine, São Paulo, SP, Brazil Bipolar Disorder Research Program, Institute of Psychiatry, Universidade de São Paulo (USP) School of Medicine, São Paulo, SP, Brazil

Lena Nabuco de Abreu Bipolar Disorder Research Program, Institute of Psychiatry, Universidade de São Paulo (USP) School of Medicine, São Paulo, SP, Brazil

Beny Lafer Bipolar Disorder Research Program, Institute of Psychiatry, Universidade de São Paulo (USP) School of Medicine, São Paulo, SP, Brazil Department of Psychiatry, Universidade de São Paulo (USP) School of Medicine, São Paulo, SP, Brazil

Table 1 - Results of the Brazilian Social Skills Inventory, by domain and by groups

\begin{tabular}{lccccccc}
\hline & \multicolumn{2}{c}{$\begin{array}{c}\text { Healthy controls } \\
(\mathbf{N}=\mathbf{3 1})\end{array}$} & \multicolumn{2}{c}{$\begin{array}{c}\text { Suicide attempters* } \\
(\mathbf{N}=\mathbf{1 2})\end{array}$} & \multicolumn{2}{c}{$\begin{array}{c}\text { Non-attempters } \\
(\mathbf{N}=\mathbf{1 6})\end{array}$} & $\begin{array}{c}\text { Covariate by age and } \\
\text { educational level }\end{array}$ \\
\cline { 2 - 8 } & Mean & SD & Mean & SD & Mean & SD & p-value \\
\hline Age & 35.30 & 6.96 & 31.00 & 7.34 & 39.60 & 11.02 & $0.032^{1}$ \\
Educational level (years) & 11.74 & 1.98 & 12.92 & 2.36 & 12.95 & 2.01 & $0.053^{1}$ \\
Intellectual level (IQ) & 97.23 & 9.50 & 100.38 & 11.96 & 97.14 & 11.97 & $0.525^{1}$ \\
Social skills (IHS) - total score & 70,00 & 25.88 & 37,50 & 25.53 & 55.00 & 32.15 & $0.007^{2}$ \\
IHS_domain 1 & 65.71 & 26.44 & 48.75 & 27.06 & 63.69 & 30.82 & $0.127^{2}$ \\
IHS_domain 2 & 56.94 & 29.48 & 47.58 & 25.64 & 53.63 & 29.18 & $0.83^{2}$ \\
IHS_domain 3 & 78.26 & 25.72 & 24.42 & 30.44 & 53.50 & 42.64 & $<0.001^{2}$ \\
IHS_domain 4 & 64.94 & 24.81 & 35.83 & 18.57 & 43.38 & 31.79 & $0.001^{2}$ \\
IHS_domain 5 & 47.42 & 24.15 & 29.50 & 30.24 & 54.25 & 30.38 & $0.041^{2}$ \\
Age & 35.30 & 6.96 & 31.00 & 7.34 & 39.60 & 11.02 & $0.032^{1}$ \\
\hline
\end{tabular}

${ }^{*}$ To indicate what group is different in comparison healthy controls (ANCOVA method)

${ }^{1}$ Kruskal Wallis method

${ }^{2}$ ANCOVA method 
Disclosures

\begin{tabular}{|c|c|c|c|c|c|c|c|}
\hline $\begin{array}{l}\text { Writing group } \\
\text { member }\end{array}$ & Employment & $\begin{array}{l}\text { Research } \\
\text { grant }^{1}\end{array}$ & $\begin{array}{l}\text { Other research grant or } \\
\text { medical continuous } \\
\text { education }\end{array}$ & $\begin{array}{l}\text { Speaker's } \\
\text { honoraria }\end{array}$ & $\begin{array}{l}\text { Ownership } \\
\text { interest }\end{array}$ & $\begin{array}{l}\text { Consultant/ } \\
\text { Advisory } \\
\text { board }\end{array}$ & Other $^{3}$ \\
\hline $\begin{array}{l}\text { Cristiana } \\
\text { Castanho de } \\
\text { Almeida Rocca }\end{array}$ & $\begin{array}{l}\text { IPq-HC- } \\
\text { FMUSP }\end{array}$ & - & - & - & - & - & - \\
\hline $\begin{array}{l}\text { Luciana } \\
\text { Gerchmann }\end{array}$ & $\begin{array}{l}\text { IPq-HC- } \\
\text { FMUSP }\end{array}$ & - & - & - & - & - & - \\
\hline $\begin{array}{l}\text { Lena Nabuco de } \\
\text { Abreu }\end{array}$ & USP & - & - & - & - & - & - \\
\hline Beny Lafer & $\mathrm{USP}^{* *}$ & $\begin{array}{l}\text { FAPESP***} \\
\text { CNPq }^{* * *}\end{array}$ & $\begin{array}{c}\text { APA/AstraZeneca Young } \\
\text { Minds in Psychiatry } \\
\text { International Awards*** }\end{array}$ & AstraZeneca* & - & - & - \\
\hline
\end{tabular}

* Modest

** Significant

*** Significant: Amounts given to the author's institution or to a colleague for research in which the author has participation, not directly to the author.

Note: IPq-HC-FMUSP = Instituto de Psiquiatria, Hospital das Clínicas, Faculdade de Medicina, Universidade de São Paulo; FAPESP = Fundação de Amparo a Pesquisa de São Paulo; CNPq = Conselho Nacional de Desenvolvimento Cientifico e Tecnológico.

For more information, see Instructions for authors.

References

1. Zalsman G, Braun M, Arendt M, Grunebaum MF, Sher L, Burke AK, Brent DA, Chaudhury SR, Mann JJ, Oquendo MA. A comparison of the medical lethality of suicide attempts in bipolar and major depressive disorders. Bipolar Disord. 2006;8(5 Pt 2):558-65.

2. de Almeida Rocca CC, de Macedo-Soares MB, Gorenstein C, Tamada RS, Issler CK, Dias RS, Schwartzmann AM, Lafer B. Social dysfunction in bipolar disorder: pilot study. Aust NZ J Psychiatry. 2008;42(8):686-92.

3. Del Prette ZAP, Del Prette A. Inventário de Habilidades Sociais (IHS-DEL PRETTE): Manual de aplicação, apuração e interpretação. São Paulo: Casa do Psicólogo; 2001. 